\title{
Análise dos trabalhos de conclusão de curso da graduação de enfermagem da universidade castelo branco 2018.1
}

Analysis of the course completion works of the castle white university nursing graduation 2018.1

Análisis de los trabajos de terminación del curso de la graduación de enfermería de la universidad blanca del castillo 2018.1

\section{RESUMO}

As instituições de ensino superior (IES) possibilitam a todo estudante uma forma de ingressar neste mundo de pesquisas. É de suma importância que haja uma coadunação entre o discente e o campo de pesquisa, pois a mesma tem a prerrogativa de gerar perfis de estudantes com a visão mais ampla sobre o assunto pesquisado.0 trabalho em questão tem como principal objetivo identificar as dificuldades dos academicos de enfermagem na elaboração dos Trabalhos de conclusão de curso, e decifra-los facilitando assim o processo de construção. Alavancando o nível intelectual do discente introduzindo-o no universo de pesquisa cientifica, centrada na busca do conhecimento e aprimoramento quanto ao patamar cientifico do profissional na área de saúde desmistificando a definição de atuação empírica até então imposta à profissão. A pesquisa surge como arte de produção, resultante de um fruto facultativo. As pesquisas dentro do campo da saúde, em especial a Enfermagem ao qual se dirige esse artigo, são de extrema relevância para a construção do conhecimento quanto discente e profissional, considerando que os níveis de conhecimento são diferenciados quanto a pesquisa e ao ensino. Este conhecimento ganho ao longo da graduação estabelece base científica que garante ao novo profissional do mercado de trabalho uma credibilidade e autoconfiança capaz de superar os estigmas sociais.
\end{abstract}

DESCRITORES: Artigo; Pesquisa de Enfermagem; Universitário.

\begin{abstract}
Higher education institutions (HEIs) provide every student with a way to enter this world of research. It is of utmost importance that there is a connection between the student and the research field since it has the prerogative of generating student profiles with a broader view on the researched subject. The work in question has as main objective to identify the difficulties of the students. nursing academics in the elaboration of the course conclusion works and decipher them thus facilitating the construction process. Leveraging the intellectual level of the student, introducing him into the universe of scientific research, centered on the search for knowledge and improvement as to the scientific level of the health professional, demystifying the definition of empirical performance previously imposed on the profession. Research emerges as a production art, resulting from an optional fruit. Research within the health field, especially Nursing to which this article is directed, is extremely relevant for the construction of knowledge in terms of students and professionals, considering that the levels of knowledge are different in terms of research and teaching. This knowledge gained during graduation establishes a scientific basis that guarantees the new professional of the job market a credibility and self-confidence capable of overcoming social stigmas.
\end{abstract}

DESCRIPTORS: Article; Nursing Research; University.

\section{RESUMEN}

Las instituciones de educación superior (IES) brindan a cada estudiante una forma de ingresar a este mundo de investigación. Es de suma importancia que exista una conexión entre el estudiante y el campo de investigación, ya que tiene la prerrogativa de generar perfiles de estudiantes con una visión más amplia sobre el tema investigado.El trabajo en cuestión tiene como principal objetivo identificar las dificultades de los estudiantes. académicos de enfermería en la elaboración de los trabajos de conclusión del curso, y descifrarlos facilitando así el proceso de construcción. Aprovechar el nivel intelectual del alumno, introducirlo en el universo de la investigación científica, centrado en la búsqueda del conocimiento y la mejora en cuanto al nivel científico del profesional de la salud, desmitificando la definición de desempeño empírico previamente impuesta a la profesión. La investigación surge como un arte de producción, resultado de una fruta opcional. La investigación dentro del campo de la salud, especialmente Enfermería a la que se dirige este artículo, es de suma relevancia para la construcción del conocimiento en términos de estudiantes y profesionales, considerando que los niveles de conocimiento son diferentes en términos de investigación y docencia. 
Este conocimiento adquirido durante la graduación establece una base científica que garantiza al nuevo profesional del mercado laboral una credibilidad y confianza en sí mismo capaz de superar los estigmas sociales.

DESCRIPTORES: Espiritualidad; Alfabetización; Autoconciencia; Embarazo.

RECEBIDO EM: 17/03/2020 APROVADO EM: 17/03/2020

\title{
Maria Regina Bernardo da Silva
}

Mestre em Saúde da Família e Docente da Universidade Castelo Branco e UNICBE.

ORCID: 0000-0002-3620-3091

\section{Francisca Regilene de Sousa de Deus}

Acadêmica de Enfermagem da Universidade Castelo Branco.

ORCID: 0000-0002-4610-1283

\section{Jéssica de Andrade Limeira}

Acadêmica de Enfermagem da Universidade Castelo Branco.

ORCID: 0000-0002-8254-0515

\author{
Halene Cristina Dias de Armada e Silva \\ Doutoranda da UERJ, Coordenadora da Cap 5.2 SMSRJ. \\ ORCID: 0000-0001-7427-781X
}

\section{Bruno Ferreira do Serrado Barbosa}

Pós-doutor UERJ, Docente da Universidade Castelo Branco, Fiscal do Coren RJ.

ORCID: 0000-0001-9942-4919

\section{Claudia da Silva de Medeiros}

Mestre em Saúde da Família e Docente da Universidade castelo Branco.

ORCID: 0000-0003-0471-0731

\section{Erica Cristina do Nascimento}

Enfermeira. Especialização em Saúde Pública e Doenças Infecciosas pela FIOCRUZ. ORCID: 0000-0001-9888-2459

\section{INTRODUÇÃO}

A enfermagem fundamentada na ciência tem se embasado nas pesquisas para alcançar novos nortes, buscando atingir mestria nos assuntos abordados. O Conselho Nacional de Desenvolvimento Científico e Tecnológico (CNPq) tem a prerrogativa de conceder condiçôes para encorajar os pesquisadores no Brasil, tendo como principais objetivos criar estratégias e agir no financiamento de pesquisas, o que de certa forma entusiasma os discentes que entram ano após ano neste ramo ${ }^{(1)}$.

Os profissionais em construção e seus orientadores ficam incumbidos de tornar palpável a condição de construção do tão aguardado projeto de término de curso $^{(2)}$.

De certa forma nota-se que as atividades de extensão e pesquisa precisam ser predominantes componentes para a reflexão do ensino neste milênio. As instituições de ensino superior (IES) corroboram com papel social de incluir o cumprimento de conhecimento social e científico, tornando este conhecimento acessível a todos, assim, o seu intuito estará em obter resultados ${ }^{(3)}$.

As IES possibilitam aos estudantes uma forma de ingressar neste mundo de pesquisas, inclusive aos que já estão concluindo e que, de certa forma, não obtiveram a oportunidade de participar de um grupo de pesquisa. Estes agora terão que executar o trabalho de conclusão de curso (TCC), com conhecimento sobre técnicas de pesquisa. É de suma importância que haja uma coadunação entre o discente e o campo de pesquisa, pois a mesma tem a prerrogativa de gerar perfis de estudantes com a visão mais ampla sobre o assunto pesquisado ${ }^{(4)}$.

Considerando que pesquisa é um trabalho árduo e único, o projeto de conclusão de curso trata-se de uma entrega por parte do discente e do orientador, se faz necessário que ambos entrem na jornada, de alguns meses e até mesmo anos, em busca do término desta obra. Com isso, cabe ao orientador ser responsável por 
levar informações que talvez ainda sejam desconhecidas pelo orientando, de forma que o envolva completamente no assunto escolhido como tema ${ }^{(5)}$.

É o orientador que, junto ao discente, fica responsável por definir o programa de estudo e pesquisa que será utilizado. O discente por vez fará a busca por documentos e avançará suas respectivas etapas necessárias, permitindo que o orientador utilize cada entrega ${ }^{(6)}$.

O fazer acadêmico chega com toda prática social, se estabelecendo em etapas e procedimentos, ao quail auxiliará o discente com suas pesquisas. As pesquisas encontram-se dividas em: revisão de literatura, que se aplica a estruturar resumos críticos de estudos sobre um tópico de um assunto de interesse, com o intuito de contextualizar o problema de pesquisa, nesta revisão é de total relevância que os dados arrecadados apontem diretamente para o objetivo da pesquisa; a revisão sistemática se caracteriza por evidenciar pesquisas cuidadosamente resumidas, buscando serem aplicadas nas respostas às questões na prática clínica, sendo conduzida por um processo rígido de seleção de material que traga produções sobre o problema estudado ${ }^{(7)}$.

Ainda pode-se incluir as pesquisas de metanálise, que utilizam a metodologia quantitativa que averigua a associação de resultados de vários estudos recentes sobre um determinado assunto. A revisão integrativa aborda resultados de pesquisas passadas, ou seja, pesquisas que já foram realizadas a fim de expor, sobretudo, as questões que norteiam e orientam a busca dessa literatura. Os dados verificados permitem que se obtenha conclusões sobre o problema abordado na pesquisa ${ }^{(8)}$.

A pesquisa é o fator determinante que qualquer projeto, quer seja ele, artigos científicos ou projeto de conclusão de curso. O seu dialeto precisa ser simples, capaz de trazer de forma simplificada o entendimento aos seus leitores. Um bom estudo, bem elaborado, e que tenha conteúdo amplo, movimento histórico e que traga, principalmente, o conhecimento que, certamente, atrairá os ledores ${ }^{(9)}$.
Ao entender um pouco mais a literatura referente à elaboração de projetos e objetos de construção de pesquisa, percebe-se a estreita relação que existe entre os dois, são tão próximas que por vezes são capazes de serem confundidas, pois seus limites acabam se chocando. As informações e direcionamentos precisam ser bem colocados visando um maior aproveitamento de quem está fazendo o uso da leitura ofertada ${ }^{(10)}$.

Conforme visualizado até este momento, a construção de um objeto de pesquisa, quer seja ele projeto de pesquisa (objeto de pesquisa) ou um TCC, requer muito mais que apenas conhecimento prévio que aborde o assunto, o que definirá o sucesso deste será o empenho direcionado, o esforço mantido junto às técnicas existentes ${ }^{(11)}$.

Portanto, nosso problema é: Quais são as dificuldades que os acadêmicos de enfermagem tiveram em relação à elaboração do seu Trabalhao de Conclusão de Curso? Objetivou-se identificar as dificuldades dos acadêmicos de enfermagem de 2018/1 de uma universidade privada na elaboração dos Trabalhos de Conclusão de Curso.

\section{METODOLOGIA}

O estudo em questão trata-se de uma pesquisa quantitava exploratória com o objetivo de identificar dificuldades encontrados na elaboração dos TCCs na graduação em enfermagem. Para a análise dos dados foi consultado o banco de TCCs de formandos do ano de 2018 do

Gráfico 1. Área de pesquisa de discentes de graduação em enfermagem de

uma universidade privada. Rio de Janeiro, RJ, Brasil, 2018-2020 primeiro semestre de uma Universidade privada, unidade Realengo, na Zona Oeste, Rio de janeiro. O curso de graduação em enfermagem a partir de 2017, no final do curso, os discentes apresentam seu TCC em formato de artigo. E os docentes e a direção da universidade têm a preocupação da formação profissional e incentivo à pesquisa. Essa pesquisa iniciou através de um projeto de extensão chamado CONSTRUCB, cujo objetivo foi de identificar as caracteristicas, tipo de metodologia, dificuldades, assuntos discutidos pelos discentes no ano de 2018 do primeiro semestre. Foram analisados os artigos que se encontravam arquivados na biblioteca da universidade. O tempo de coleta, tratamento e análise de dados foi de setembro de 2018 a janeiro de 2020. O acervo contabilizou o somatório de 20 artigos. Por se tratar de dados disponíveis em base de dados de acesso público, o estudo dispensa a submissão ao Comitê de Ética em Pesquisa.

\section{RESULTADOS}

De acordo com os resultados acima, as áreas de pesquisa encontradas nos artigos foram: Saúde Coletiva 01 (5\%), Profissional 01 (5\%), Educacional 05 (25\%), Assistencial 10 (50\%), Tecnológico 0 (nenhum) e Saude Mental 03 (15\%).

Mediante análise dos dados referidos, pode-se observar uma predominância quanto aos artigos na área assistencial, logo em seguida a área educacional, e também observou-se na área de saúde mental onde os discentes têm

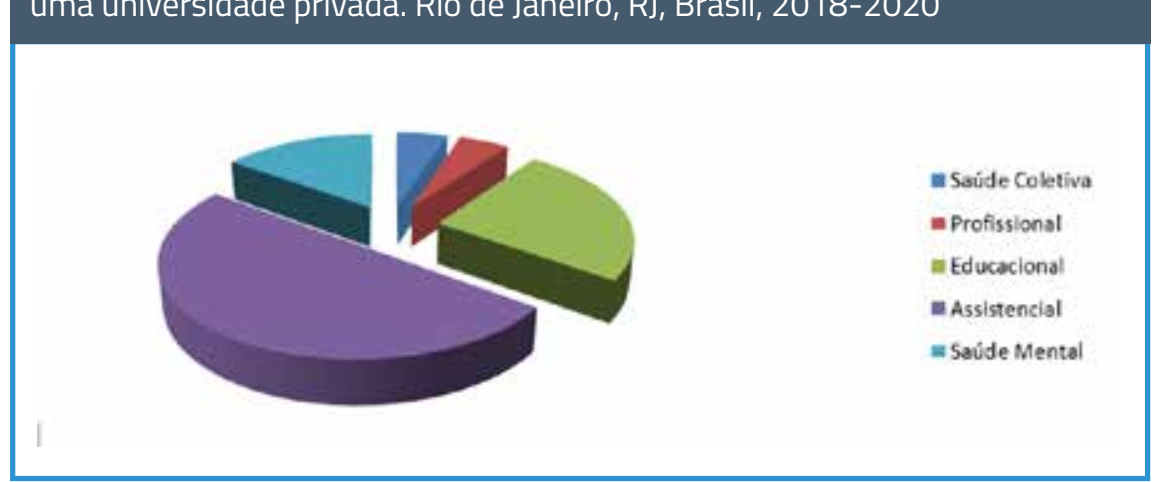




\section{artigo}

Bernardo da Silva, M.R.; Deus, F.R.S.; Limeira, J.A.; Armada e Silva, H.C.D.; Barbosa, B.F.S.S; Medeiros, C.S.; Nascimento, E.C.;

Análise dos trabalhos de conclusão de curso da graduação de enfermagem da universidade castelo branco 2018.1

tido alguma preocupação em relação às doenças mentais e è enfermagem. Observa-se, assim, que estas áreas são as de maior interesse dos discentes, talvez pelo contato direto e in- tenso com a educação e suas atualizações, ou devido à curiosidade do conhecimento com a área assistencial e doenças ocupacionais e mentais da enfermagem.
Gráfico 2. As metodologias usadas pelos discentes de graduação em enfermagem de uma universidade privada. Rio de Janeiro, RJ, Brasil, 2018-2020

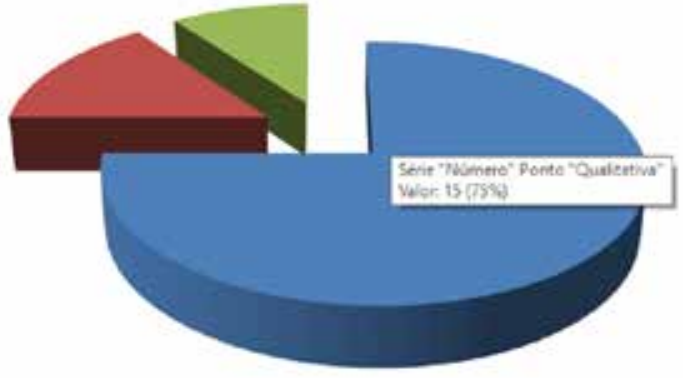

Eualitativo

Euantitativa

= Quali-Quantitativa

Gráfico 3. Tipos de estudo usados pelos discentes de graduação em enfermagem de uma universidade privada. Rio de Janeiro, RJ, Brasil, 2018-2020

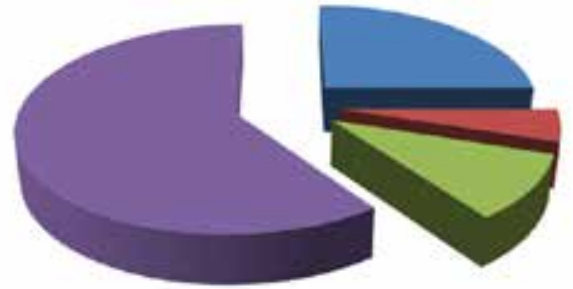

Descritivo

Exploratỡilo

Descritivo e Exploratório

Näo Especificado

Gráfico 4. Tipos de instrumentos de pesquisa usados pelos discentes de graduação em enfermagem de uma universidade privada. Rio de Janeiro, RJ, Brasil, 2018-2020

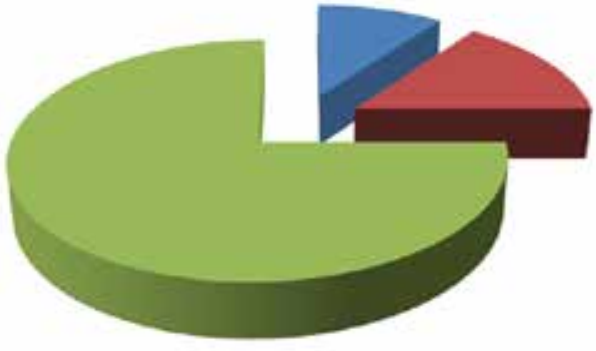

Entrevista

equestiondrio

=Levantamento bibliográfico
A metodologia qualitativa foi a mais utilizada $15(75 \%)$ nas pesquisas, em seguida a quantitativa $03(15 \%)$ e a quali-quantitava $02(10 \%)$.

Os artigos científicos têm como propósito na área da saúde analisar, orientar e aprimorar as práticas. Ao longo dos anos, as práticas aplicadas nos primórdios foram atualizadas e ganharam embasamento científico, sempre com enfoque principal na redução de danos ao paciente. Podemos presumir que a diferença significativa encontrada nos dados acima dá-se mediante a busca de elevação da qualidade das práticas e conhecimentos inerentes à Enfermagem.

De acordo com a pesquisa, os estudos utilizados foram os não especificados 12 (60\%), descritivos 05 (25\%), em seguida descritivo e exploratório 03 (15\%) e os do tipo experimental e observacional não foram encontrados.

Conforme gráfico acima, podemos constatar que a praticidade sobre o estudo experimental não foi explorada, pois demanda tempo e, na maioria das vezes, há pouco conhecimento por parte dos discentes e dificuldade em pesquisar. Conclui-se que o docente precisa explorar este tipo de pesquisa, para agregar conhecimento aos discentes.

De acordo com os tipos de instrumentos encontrados, a revisão de literatura apresentou-se em 15 (75\%), em seguida, questionário 03 (15\%) e entrevista 02 (10\%). A revisão de literatura dentro deste aparato foi o instrumento de pesquisa mais utilizado, talvez devido a fácil acessibilidade aos dados e às implicações burocráticas que os outros métodos necessitam, como por exemplo, a autorização do Comitê de Ética, ou ainda a disponibilidade, tempo e expectativa que a pesquisa possa custar. Considerando que, na maioria das vezes, os alunos investem na construção do seu TCC em períodos bem avançados ao término, disponibilizando, assim, pouco tempo para elaboração. Além do tempo, a dificuldade de pesquisar também contribui para a não elaboração de pesquisas de campo.

Ao avaliarmos os sujeitos do estudo, o Enfermeiro e a Mulher surgem em destaque, evidenciando, assim, o interesse dos discen- 
tes. Nota-se que, ao longo do seu percurso, esses surgiram como parâmetros para sua base de dados. O direcionamento das suas pesquisas nos revela o interesse que está surgindo até o final de sua graduação e após.

Através dos dados identificados na pesquisa, avaliou-se o déficit, havendo necessidade de maior interferência pelos docentes, visto que os discentes em questão possuem inexperiência na prática, é importante ressaltar que são iniciantes, como podemos chamar são "novatos" nessa arte de escrever literatura. Devido a isso, observam-se escolhas que podem ser classificadas como mais fáceis, pois exigem menor disponibilidade de tempo e locomoção por parte do pesquisador.

Durante o processo de formação, o discente deveria explorar todas as formas de pesquisa, a fim de expandir seu conhecimento e, até mesmo, a sua capacidade de alcançar novos horizontes, considerando que após a formação ele terá que realizar essas tarefas so-

Tabela 1. Sujeitos dos estudos usados pelos discentes de graduação em enfermagem de uma universidade privada. Rio de Janeiro, RJ, Brasil, 2018-2020

\section{Sujeito dos Estudos}

Acadêmicos de Enfermagem

Adultos

Adolescente

Enfermeiro

Equipe de Enfermagem

Mulher

Paciente Psiquiátrico

Total zinhos e solucionar quaisquer problemáticas do cotidiano.

Considerando que a pesquisa na carreira iniciante do discente é uma constante construção para o currículo Lattes, observa-se que é de total relevância investir o mais precoce possível, acarretando chances de obter o máximo de títulos possíveis para o início de sua carreira de formado ${ }^{(12)}$.

Os discentes que têm maior desenvolvimento neste ciclo apresentaram interesses mais amplos e diversificados, um bom sinal para a adaptação ao mundo do trabalho volátil ${ }^{(13)}$.

A construção da identidade do acadêmico é construída através da sua trajetória na graduação universitária, ou seja, é preciso que este se envolva em eventos, projetos ou práticas que sejam de relevância para seu período dentro da universidade ${ }^{(14)}$.

\section{DISCUSSÃO}

$\begin{array}{cc}\text { Número } & \% \\ 02 & 10 \% \\ 01 & 5 \% \\ 01 & 5 \% \\ 06 & 30 \% \\ 02 & 10 \% \\ 07 & 35 \% \\ 01 & 5 \% \\ 20 & 100 \%\end{array}$

Tabela 2. Cenário de pesquisa usado pelos discentes de graduação em enfermagem de uma universidade privada. Rio de Janeiro, RJ, Brasil, 2018-2020

\section{Cenário da Pesquisa}

Gerência Regional de saúde

Hospital

Programa de Saúde da Familia

Programa de Saúde na Escola

Universidade

CAPS

Não especificado

Total

\section{Número}

01

07

02

01

02

01

06

20

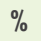

$5 \%$

$35 \%$

$10 \%$

$5 \%$

$10 \%$

$5 \%$

$30 \%$

$100 \%$
O trabalho de pesquisa, quando bem elaborado, qualifica seus resultados, possibilitando identificar lacunas no conhecimento em relação aos temas abordados em forma de estudo. Identificar possíveis necessidades futuras de pesquisa é um passo crucial para o acadêmico, bem como revelar questões que abordem áreas de foco, marcos conceituais e, até mesmo, teóricos. A pesquisa surge como arte de produção, resultante de um fruto facultativo ${ }^{(15)}$.

As pesquisas dentro do campo da saúde, em especial a Enfermagem, ao qual se dirige esse artigo, são de extrema relevância para a construção do conhecimento quanto discente e profissional, considerando que os níveis de conhecimento são diferentes quanto pesquisa e quanto ao ensino. Estabelece-se uma necessidade de maior amplitude de conhecimento dos temas a serem pesquisados e maior conexão do discente com o docente na pesquisa.

Ao longo da graduação estabelece-se o conhecimento científico, que garante ao novo profissional no mercado de trabalho credibilidade e autoconfiança, capazes de superar os estigmas sociais $^{(16)}$.

$\mathrm{Na}$ área da saúde, todas as ações e intervenções precisam ser norteadas por profissionais, usuários e gestores, garantindo a equidade, neste caso, antes de programar qualquer procedimento novo ou instalar uma informação no mercado de saúde, são feitos apanhados de informações, ou seja, torna-se inviável a identificação de dados fidedignos sem antes ter tido uma anamnese de qualidade ${ }^{(17)}$.

\section{CONSIDERAÇÕES FINAIS}

Este artigo tem como objetivo investigar e analisar a partir de uma perspectiva sistêmica, as concepções, estratégias, motivações, abordagens, práticas, efeitos e resultados dos TCCs efetivamente concluídos do curso de Enfermagem, que se encontram à disposição, servindo como um objeto de pesquisa.

Conclui-se que os projetos de extensão colaboram para essa estruturação e formação do TCC, essa base é o que possibilita uma compreensão de suas relações entre o escritor e seus leitores. 


\section{artigo}

Bernardo da Silva, M.R.; Deus, F.R.S.; Limeira, J.A.; Armada e Silva, H.C.D.; Barbosa, B.F.S.S; Medeiros, C.S.; Nascimento, E.C.;

Análise dos trabalhos de conclusão de curso da graduação de enfermagem da universidade castelo branco 2018.1

Importante ressaltar que, além da vontade e disposição dos discentes, faz-se importante considerar as dificuldades encontradas dentro do campus universitário e ainda no espaço de pesquisa. Com isso, é tão importante o discente identificar seu espaço e suas preferências para que a pesquisa se torne algo agradável.
Observou-se que as dificuldades encontradas para realização dos TCCs estão relacionadas à pouca leitura, não saber o tema definido, não saber pesquisar e pouco tempo para a pesquisa.

A graduação constitui-se como principal necessidade, sendo fundamental para a construção do conhecimento, considerando que é neste momento que pesquisadores se reconhecem e são reconhecidos, é com este conhecimento que este trilhará os caminhos iniciais de pesquisa que, em breve, lhe serão transformados no TCC. Neste intervalo, durante sua estadia na universidade, o discente aprende a valorizar a cultura do consumo e produção de pesquisa.

\section{REFERÊNCIAS}

1. Erdeman, et al; Vislumbrando o significado da iniciação científica a partir do graduando de enfermagem. Esc Anna Nery Rev Enferm [Internet]. 2010 jan-mar [acesso em 06 set 2018]; 14(1):26-32. Disponível em: http://www.redalyc.org/ pdf/1277/127712632005.pdf.

2. Real LMC, Corbellini S. Trabalho de Conclusão de Curso (TCC) em um Curso de Graduação Modalidade EAD: uma Proposta Cooperativa construída à distância Universidade Federal do Rio Grande do Sul [Internet]. 2011 jul [acesso em 24 fev 2020];9(1). Disponivel em: https://www.seer.ufrgs.br/renote/article/ view/21909/12711

3. Nunes ALDPF, Silva MBDC. A extensão universitária no ensino superior e a sociedade. Mal-Estar e Sociedade [Internet]. 2011 jul./dez. [acesso em 10 set 2018];IV(7):119-133. Disponivel em: http://revista.uemg.br/index.php/malestar/article/view/60/89 .

4. Medeiros MC, Rocha FAF, Silva RCL, Danjour MF. Dificuldades do processo de orientação em trabalhos de conclusão de curso (TCC): um estudo com os docentes do curso de administração de uma instituição privada de ensino superior. Instituto Federal do Rio Grande do Norte. [acesso em 23 fev 2010]. Disponível em: http://www2.ifrn.edu.br/ojs/index.php/HOLOS/article/viw/1011/1147e.

5. Chaer G, Diniz RRP, Ribeiro EA. A técnica do questionário na pesquisa educacional. Evidência [Internet]. 2011 [acesso em 06 set 2018];7(7):251-266. Disponivel em: http://www.uniaraxa. edu.br/ojs/index.php/evidencia/article/view/201/187.

6. Alves LC, Urquiza MF, Roland CEDF. Gestor de tcc: desenvolvimento de sistema para gestão de trabalhos acadêmicos. Rev Eletr Comp Aplic [Internet]. 2016 [acesso em 24 fev 2020];7(1). Disponível em: http://periodicos.unifacef.com.br/index.php/ resiget/article/view/1148/886.

7. Crossetti MDGO. Revisão integrativa de pesquisa na Enfermagem. O rigor cientifica que Ihe é exigido. Rev Gaúcha Enferm [Internet]. 2012 jun [acesso em 15 set 2018];33(2):8-9. Disponivel em: https://www.lume.ufrgs.br/bitstream/handle.

8. Stake RE, Reis K. Métodos de pesquisa, pesquisa qualitativa, estudando como as coisas funcionam. Rio de Janeiro: ArtMED Editora; 2010.

9. Lima TCSL, Mioto RCT. Procedimentos metodológicos na construção do conhecimento científico: a pesquisa bibliográfica. Rev katálysis [Internet]. 2007 [acesso em 24 fev 2020]10(Spe). Disponivel em: http://www.scielo.br/scielo.php?pid=S1414-49802 007000300004\&script=sci_arttext.

10. Ludke M. Universidade, escola de educação básica e o problema do estágio na formação de professores. Revista Brasileira de Pesquisa sobre formação docente. Form. Doc. [Internet]. 2009 ago./dez. [acesso em 21 set 2018];01(01):95-108. Disponivel em http://formacaodocente.autenticaeditora.com.br.

11. Araújo J, Pimenta AA, Costa S. A proposta de um quadro norteador de pesquisa como exercício de construção do objeto de estudo. Interações [Internet]. 2015 jan./jun. [acesso em 30 out 2018];16(1):175-188. Disponivel em: http://www.scielo.br/pdf/ inter/v16n1/1518-7012-inter-16-01-0175.pdf.

12. Antunes AL, Rodrigues PAM, Brandão Z. Hierarquias acadêmicas na pesquisa em educação. Educ. Pesqui [Internet]. 2019 [acesso em 30 out 2018];45. Disponivel em: http://www. scielo.br/scielo.php?script=sci_arttext\&pid=S1517-970220190 00100604\&lang=pt.

13. Leal MS, Fracalozzi NMN. Maturidade profissional como diagnóstico de necessidades de educação para carreira. Psicologia Argumento [Internet]. 2019 [acesso em 20 fev 2020];37(97). Disponivel em: https://periodicos.pucpr.br/index.php/psicologiaargumento/article/view/25907.

14. Bezerra B. Letramentos acadêmicos e construção da identidade: a produção do artigo científico por alunos de graduação. Ling. (dis)curso [Internet]. 2015 jan./abr. [acesso em 25 fev 2020];15(1). Disponivel em: http://www.scielo.br/scielo. php?script=sci_arttext\&pid=S1518-76322015000100061\&la $\mathrm{ng}=\mathrm{pt}$.

15. Ludke M. O professor, seu saber e sua pesquisa". In: Revista Educação e Sociedade [Internet]. 2008 abr [acesso em 30 out 2019];XXII(74). Disponivel em: htpp://www.scielo.br/pdf/es/ v22n74/a06v2274.pdf.

16. Giacchero KG, Miasso Al. A produção científica na graduação em enfermagem (1997 a 2004): análise crítica. Rev. Eletr. Enf. [Internet]. 2009 [acesso em 02 nov 2019]; 8(3):431-40. Disponivel em: http://www.fen.ufg.br/revista/revista8 3/pdf/ v8n3a14.pdf.

17. Antunes EDD, Brunetta N, Demarco DJ, Pinheiro IA. Desafios na construção do trabalho de conclusão de curso de especialização em negociação coletiva/ modalidade a distância. Revista CINTED - UFRGS [Internet]. $2011 \mathrm{dez}$ [acesso em $20 \mathrm{dez}$ 2019];9(2). Disponivel em: https://seer.ufrgs.br/renote/article/ view/25140. 